\title{
Transcriptomic profiles of Dunaliella salina in response to hypersaline stress
}

\author{
Qinghua He ${ }^{1 \dagger}$, Yaqiu Lin ${ }^{1 \dagger}$, Hong Tan ${ }^{2}$, Yu Zhour ${ }^{2}$ Yongli Wen², Jiajia Gan ${ }^{1}$, Ruiwen Li ${ }^{3^{*}}$ and Qinglian Zhang ${ }^{4^{*}}$ (D)
}

\begin{abstract}
Background: Dunaliella salina is a good model organism for studying salt stress. In order to have a global understanding of the expression profiles of Dunaliella salina in response to hypersaline stress, we performed quantitative transcriptomic analysis of Dunaliella salina under hypersaline stress (2.5 M NaCl) of different time duration by the second and third generation sequencing method.

Results: Functional enrichment of the up-regulated genes was used to analyze the expression profiles. The enrichment of photosynthesis was observed, accompanied by enrichments of carbon fixation, pigment biosynthetic process and heme biosynthetic process, which also imply the enhancement of photosynthesis. Genes responsible for starch hydrolysis and glycerol synthesis were significantly up-regulated. The enrichment of biosynthesis of unsaturated fatty acids implies the plasma membrane undergoes changes in desaturation pattern. The enrichment of endocytosis implies the degradation of plasma membrane and might help the synthesis of new glycerophospholipid with unsaturated fatty acids. Co-enrichments of protein synthesis and degradation imply a higher protein turnover rate. The enrichments of spliceosome and protein processing in endoplasmic reticulum imply the enhancement of regulations at post-transcriptional and post-translational level. No up-regulation of any $\mathrm{Na}^{+}$or $\mathrm{Cl}^{-}$channels or transporters was detected, which implies that the extra exclusion of the ions by membrane transporters is possibly not needed. Voltage gated $\mathrm{Na}^{+}$and $\mathrm{Cl}^{-}$channels, mechanosensitive ion channel are possible signal receptors of salt stress, and $\mathrm{Ca}^{2+}$ and MAP kinase pathways might play a role in signal transduction.

Conclusion: At global transcriptomic level, the response of Dunaliella salina to hypersaline stress is a systematic work, possibly involving enhancements of photosynthesis, carbon fixation, and heme biosynthetic process, acceleration of protein turnover, spliceosome, protein processing in endoplasmic reticulum, and endocytosis, as well as degradation of starch, synthesis of glycerol, membrane lipid desaturation. Altogether, the changes of these biological processes occurred at trancriptomic level will help understand how a new intracellular balance achieved in Dunaliella salina to adapt to hypersaline environment, which are worth being confirmed at the physiological levels.
\end{abstract}

Keywords: Dunaliella salina, Salt stress, Glycerol, Transcriptomics analysis, Third-generation sequencing, Secondgeneration sequencing

\section{Background}

Dunaliella is an extremely halotolerant, unicellular, green algae, which is unique in its remarkable ability to survive in media containing $\mathrm{NaCl}$ at a wide range of concentrations, from about $0.05 \mathrm{M}$ to saturation (around $5.5 \mathrm{M})$ [1]. This character makes it a good model

\footnotetext{
* Correspondence: liruiwen0001@163.com; qlzhang80@163.com

${ }^{\dagger}$ Qinghua He and Yaqiu Lin contributed equally to this work.

${ }^{3}$ Reproductive and endocrine laboratory, Chengdu Woman-Child Central

Hospital, Chengdu 610051, People's Republic of China

${ }^{4}$ School of Laboratory Medicine, Chengdu Medical College, Chengdu 610500,

People's Republic of China

Full list of author information is available at the end of the article
}

organism for studying salt tolerance. Studies on salt tolerance of Dunaliella began from 60s last century, and big progresses were made from 70 s to 90 s. First, high concentration of intracellular glycerol was found to be the main contributor for osmotic balance across plasma membrane [2]. Second, a glycerol metabolism cycle in Dunaliella was proposed, that is, for glycerol synthesis, dihydroxyacetone phosphate (DHAP) from glycolysis is converted to glycerol-3-phosphate by glycerol-3phosphate dehydrogenase (GPDH), then gycerol-3phosphate is converted to glycerol by glycerol-3phosphate phosphatase; and for glycerol dissimilation, 
glycerol is converted to dihydroxyacetone by glycerol dehydrogenase, and then dihydroxyacetone is converted to DHAP by dihydroxyacetone kinase [3]. As the key enzyme in the pathway, GPDH was extensively studied $[4,5]$. Third, the $\mathrm{Na}^{+} / \mathrm{H}^{+}$antiporter activity was detected in plasma membrane and was thought to function as exclusion of $\mathrm{Na}^{+}$in vivo [6, 7].

In twenty-first century, proteomic methods were used to understand the molecular mechanism of salt tolerance at omics level. Proteins such as transferrin, carbonic anhydrases, $\mathrm{Na}^{+} / \mathrm{H}^{+}$antiporter, fatty acid elongase, GPDH, small GTP-binding protein and tubulin were found upregulated significantly under salt stress. These proteins can be classified in carbon assimilation, energy production, transporters, signal transduction, protein synthesis and cell defense $[8,9]$. However, due to the limitation of the two-dimensional electrophoresis, the information obtained from this technique is limited $[8,9]$, with detected number of differently expressed proteins below 100, of which only about $60 \%$ can be annotated.

Compared with proteomic approaches, transcriptomic methods are more reproducible, sensitive with higher genome coverage. A transcrtiptome of 17,845 transcripts was reported when Dunaliella tertiolecta was investigated to identify the pathways and genes involved in lipid synthesis under nitrogen stress, which covers about 97\% of the core eukaryotic genes (CEGs) [10-12]. Hong et al. reported the transcriptome of Dunaliella salina at different phases of their growth cycle (30d, 80d, 120d), but no transcriptome under salt stress was reported [13]. Alkayal reported the expressed sequence tag (EST) profiling of Dunaliella salina after $5 \mathrm{~h}$ of hypersaline shock, in which a transcriptome of 1401 unique transcripts was reported and the annotated transcripts can be classified into protein synthesis, energy, primary metabolism and protein fate [14]. However, no transcriptome before salt stress was generated, so there was no comparison to present the underlying changes during this shock period. In order to have a better understanding of how Dunaliella salina responds to hypersaline shock at transcriptomic level, the second and the third generation sequencing were used to generate the transcriptome of Dunaliella salina at different duration time under stress. Because intracellular glycerol synthesis accomplished in about $2 \mathrm{~h}$ after hypersaline shock $[15,16]$, we reported the transcriptomic profiles on time duration of $0.5-\mathrm{h}, 1-$ $\mathrm{h}$ and 2-h under hypersaline stress and the profiles were compared with those before stress.

\section{Results}

Data quality and sequences annotation

To obtain high quality sequence data, total RNAs of high quality were extracted (not shown). After the second generation sequencing, each library gave high quality clean reads with Q20\% ranging from 97.21 to 98 with error rate about $0.01 \%$ (Additional file 1: Table S1). The GC content is about $56.5 \%$, which is close to Dunaliella salina (CCAP19/18) reported [17] . The number of the named "full length transcripts" generated from the third generation sequencing was 43,864, ranging from 242 to $8978 \mathrm{bp}$ in length with a mean length of $1009 \mathrm{bp}$ and median length of $918 \mathrm{bp}$. About $80 \%$ transcripts of them are in the length range of 500 to $2000 \mathrm{bp}$ (Additional file 1: Table S2). By ORF analysis, among the 43,864 transcripts, 35,175 transcripts are classified into coding sequences and 8689 transcripts are classified into long non-coding sequences. Among the 35,175 coding sequences, 29,071 sequences are annotated and 6104 sequences cannot be annotated so far. In order to estimate the coverage of the transcriptome, transcripts hit the same gene (the same sequence ID) in Nr, Nt or SwissProt database are defined as the splice variants generated by alternative splicing from a single gene. By this method isoenzymes and artificially spliced sequences are also excluded. Finally 9256 individual genes from the 29,071 transcripts are generated. Genome sequencing of Dunaliella salina (CCAP19/18) and Chlamydomonas reinhardtii predicted 16,697 and 17,741 loci containing protein-coding transcripts respectively $[17,18]$. Compared with the predicted numbers of gene loci of the two green algae, 9256 is a rather high number, since many genes aren't expressed and their mRNAs can't be detected. Furthermore, approximately $87.1 \%$ of the core eukaryotic genes (CEGs) were identified from the 9256 individual genes by sequence similarity search which suggests a rather high coverage of the Dunaliella salina transcriptome.

\section{General pattern of gene expression}

Based on gene expression value, clustering analysis was performed (Additional file 2: Figure S1), we can see the similarities of the expression patterns of the samples with good repeatability in the same group (the same stress time).

While compared with the 0-h of stress (no salt stress was applied), the number of differentially expressed genes increased with the increasing of stress duration time (Fig. 1). The number of up-regulated genes increases from 569 on 0.5 -h of stress to 915 on 1-h of stress, and then to 3071 on 2 -h of stress. On the other hand, the number of down-regulated genes increases from 513 on 0.5 -h stress to 810 on 1 -h stress, and then to 2580 on 2 -h stress.

In order to have an overall understanding of the upregulated genes under salt stress, functional enrichments were performed by GO (gene ontology) (Table 1). On 0.5$\mathrm{h}$ of stress, carboxylic acid biosynthetic process, cellular lipid metabolic process, carbohydrate metabolic process, response to temperature stimulus, photosynthesis (light 



Fig. 1 Volcano Plot of the differentially expressed genes. The differentially expressed genes were generated by comparing the gene expression values under stress of different time duration $(0.5 \mathrm{~h}, 1 \mathrm{~h}, 2 \mathrm{~h})$ with that of control $(0 \mathrm{~h})$. $\mathbf{a}$ the comparison of 0.5 - $\mathrm{h}$ of stress with that of 0 -h of stress; $\mathbf{b}$ the comparison of 1-h of stress with that of 0-h of stress; $\mathbf{c}$ the comparison of 2-h of stress with that of 0-h of stress; the number of upregulated genes increased constantly with the increasing of stress duration time, the number of down-regulated genes also increased constantly with the increasing of stress duration time

harvesting), photosynthesis (light reaction), cofactor metabolic process, pigment biosynthetic process, and tetrapyrrole biosynthetic process are significantly enriched. On 1$h$ of stress, protein folding and DNA replication are included in the list of significantly enriched biological processes, cellular lipid metabolic process and response to temperature stimulus are enriched but not statistically significant, while photosynthesis is excluded due to rapid decreasing of gene number (Table 2). On 2-h of stress, new terms such as macromolecule modification, cellular catabolic process, cell redox homeostasis, reproductive process, and ferrous iron transport are significantly enriched, while transcription (DNA-templated) is enriched, but not statistically significant. The terms enriched on 1-h of stress, such as carboxylic acid metabolic process, cellular lipid metabolic process, carbohydrate metabolic process, response to temperature stimulus, cofactor metabolic process, protein folding, and DNA replication, are also enriched and show a quick increasing of the gene numbers compared with that of 1-h of stress. These biological processes are not statistically significant due to the rapid increasing of the number of the total upregulated genes, but they are still worth focusing on. In general, the significantly enriched biological processes can be classified into photosynthesis, carbohydrate metabolism, lipid metabolism, and amino acids and protein metabolism. We focused on analyzing these biological processes in the following sections.

On the other hand, the functional enrichment of the down-regulated genes was also performed by GO (Additional file 1: Table S3). On 0.5 -h of stress, no terms were significantly enriched, but carbohydrate binding and protein binding were worth focusing on since the numbers of down-regulated genes involved are large. On 1-h of stress, DNA metabolic process, protein binding, cytoskeleton, glycoprotein biosynthetic process, glycosaminoglycan biosynthetic process, and dynein complex were significantly enriched. On 2-h of stress, more GO terms were significantly enriched beside the terms enriched on 1-h of stress, these terms include transferase activity, protein modification process, regulation of RNA biosynthetic process, response to nitrate, inorganic anion transport, lipid transport, DNA integration, autophagy, and GTPase activator activity. From the point of gene numbers, we can see that the down-regulated genes are mainly involved in protein binding, transferase activity, protein modification process, DNA metabolic process, regulation of RNA biosynthetic process, and cytoskeleton. These terms are also important for understanding the hypersaline stress of Dunaliella salina, however, this paper only focuses on the analysis of the terms enriched by the up-regulated genes.

\section{Photosynthesis}

On the 0.5 -h of stress, photosynthesis-light reaction and photosynthesis-light harvesting are significantly enriched by $\mathrm{GO}$ analysis on up-regulated genes, which implies the enhancement of photosynthesis. In time course, most genes are highly expressed on $0.5-\mathrm{h}$, decreased a little on $1-\mathrm{h}$, and then decreased to low levels even lower than that of 0 -h. The expression pattern is like a pulse style and most peaks of gene expression are induced on or before 0.5-h of stress (Fig. 2). Many genes of Chlorophyll $\mathrm{a}-\mathrm{b}$ binding proteins show pulse expression patterns, such as Chlorophyll a-b binding protein of LHCII type I, Chlorophyll $\mathrm{a}-\mathrm{b}$ binding protein type 1 member F3, Chlorophyll a-b binding protein $\mathrm{P} 4$, and Chlorophyll a-b binding protein CP29 et al.. Some of the genes show high expression on 2-h of stress, including ATPdependent zinc metalloprotease FTSH 2, Photosystem II repair protein PSB27-H1, D-amino-acid transaminase, and Photosystem II protein D1. A few genes show a decreasing of expression, including Protein TIC 20-II, Oxygen-evolving enhancer protein, and DNA-binding 
Table 1 Main biological processes significant enriched from the up-regulated genes

\begin{tabular}{|c|c|c|}
\hline GO_accession & Description & Number of Genes involved \\
\hline \multicolumn{3}{|l|}{$0.5 \mathrm{~h} \mathrm{VS} 0 \mathrm{~h}$} \\
\hline GO:0046394 & carboxylic acid biosynthetic process & 29 \\
\hline GO:0044255 & cellular lipid metabolic process & 26 \\
\hline GO:0005975 & carbohydrate metabolic process & 44 \\
\hline GO:0009266 & response to temperature stimulus & 12 \\
\hline GO:0009765 & photosynthesis, light harvesting & 35 \\
\hline GO:0019684 & photosynthesis, light reaction & 38 \\
\hline GO:0051186 & cofactor metabolic process & 52 \\
\hline GO:0046148 & pigment biosynthetic process & 24 \\
\hline GO:0033014 & tetrapyrrole biosynthetic process & 27 \\
\hline \multicolumn{3}{|l|}{$1 \mathrm{~h}$ VS $0 \mathrm{~h}$} \\
\hline GO:0019752 & carboxylic acid metabolic process & 100 \\
\hline GO:0044255 & cellular lipid metabolic process ${ }^{a}$ & 36 \\
\hline GO:0005975 & carbohydrate metabolic process & 73 \\
\hline GO:0009266 & response to temperature stimulus ${ }^{a}$ & 14 \\
\hline GO:0051186 & cofactor metabolic process & 74 \\
\hline GO:0046148 & pigment biosynthetic process & 28 \\
\hline GO:0033014 & tetrapyrrole biosynthetic process & 31 \\
\hline GO:0006457 & protein folding & 26 \\
\hline GO:0006260 & DNA replication & 28 \\
\hline \multicolumn{3}{|l|}{$2 \mathrm{hVSOh}$} \\
\hline GO:0019752 & carboxylic acid metabolic process $^{\mathrm{a}}$ & 230 \\
\hline GO:0044255 & cellular lipid metabolic process $^{\mathrm{a}}$ & 61 \\
\hline GO:0005975 & carbohydrate metabolic process & 133 \\
\hline GO:0009266 & response to temperature stimulus ${ }^{a}$ & 23 \\
\hline GO:0051186 & cofactor metabolic process $^{a}$ & 127 \\
\hline GO:0006457 & protein folding & 48 \\
\hline GO:0006260 & DNA replication ${ }^{a}$ & 57 \\
\hline GO:0006351 & transcription, DNA-templated ${ }^{a}$ & 191 \\
\hline GO:0043412 & macromolecule modification & 134 \\
\hline GO:0044248 & cellular catabolic process & 79 \\
\hline GO:0045454 & cell redox homeostasis & 32 \\
\hline GO:0022414 & reproductive process & 30 \\
\hline GO:0015684 & ferrous iron transport & 13 \\
\hline
\end{tabular}

anot significantly enriched

$11 \mathrm{kDa}$ phosphoprotein (Fig. 2). Chlorophyll biosynthetic process is also enriched, which indicates the synthesis of photosynthetic pigments to enhance photosynthesis (Table 2). This is consistent with previous study [9]. With the stress going on, the gene numbers of photosynthesis-light reaction and photosynthesis-light harvesting decreased (Table 2), while the gene number of carbon fixation constantly increased, from 12 on $0.5-\mathrm{h}$ to 25 on $1-\mathrm{h}$, and to 39 on 2 -h of stress (Table 2, Additional file 2: Figure S2), key genes such as carbonic anhydrase and rubisco activase are significantly upregulated (Additional file 1: Table S4). Compared with the decreased gene number of photosynthesis-light reaction and photosynthesis-light harvesting, the constantly increased gene number of carbon fixation indicates that these biological processes may be controlled by different signaling pathways.

With the stress going on, the gene number of chlorophyll biosynthetic process decreased, while the gene number of tetrapyrrole biosynthetic process remained 
Table 2 Enrichment of photosynthesis and photosynthetic pigments related terms

\begin{tabular}{|c|c|c|c|c|}
\hline \multirow[t]{3}{*}{ GO_ID } & \multirow[t]{3}{*}{ GO_Term } & \multicolumn{3}{|l|}{ Samples } \\
\hline & & $0.5 \mathrm{~h}$ & $1 \mathrm{~h}$ & $2 \mathrm{~h}$ \\
\hline & & Number of Genes involved & Number of Genes involved & Number of Genes involved \\
\hline GO:0015979 & photosynthesis & 50 & 40 & 31 \\
\hline GO:0009765 & photosynthesis, light harvesting & $35^{\mathrm{a}}$ & 18 & 0 \\
\hline GO:0019684 & photosynthesis, light reaction & $38^{\mathrm{a}}$ & 21 & 6 \\
\hline GO:0033014 & tetrapyrrole biosynthetic process & $27^{\mathrm{a}}$ & $31^{\mathrm{a}}$ & 27 \\
\hline GO:0015995 & chlorophyll biosynthetic process & 9 & 8 & 2 \\
\hline GO:0006783 & heme biosynthetic process & $11^{\mathrm{a}}$ & $15^{\mathrm{a}}$ & 22 \\
\hline GO:0046148 & pigment biosynthetic process & $28^{\mathrm{a}}$ & $33^{\mathrm{a}}$ & 48 \\
\hline KO_ID & KO_term & Number of Genes involved & Number of Genes involved & Number of Genes involved \\
\hline ko00710 & carbon fixation in photosynthetic organisms & 12 & 25 & 39 \\
\hline
\end{tabular}

${ }^{\mathrm{a}}$ indicates significantly enriched

stable and the gene number of heme biosynthetic process kept increasing (Table 2). The increasing of gene number of heme biosynthetic process and the decreasing of gene number of chlorophyll biosynthetic process together resulted in the stableness of gene number of tetrapyrrole biosynthetic process since the latter is the father term of the former two. This is consistent with the result of heat-map analysis, of which some genes show pulse expression pattern, these genes are clustered to chlorophyll biosynthetic process, while some genes show high expression values on 2 -h of stress, these genes are clustered to heme biosynthetic process (Additional file 2: Figure S3). The significant enrichment of tetrapyrrole biosynthetic process and heme biosynthetic process on $0.5-\mathrm{h}$ and $1-\mathrm{h}$ of stress are very interesting. In plants and algae, tetrapyrroles are plastid signals demonstrated to regulate nuclear gene expression [19-22]. Heme signaling also appears to play a role in starch biosynthesis and drought tolerance in plants [23, 24]. We see the constant increasing of gene number of heme biosynthetic process with the increasing of stress time, while large amount of signal molecules are usually not needed, so the constant increasing gene number of heme synthesis could be for the synthesis of heme-containing enzymes, such as catalase and ascorbate peroxidase, which play important roles in detoxification of reactive oxygen species (ROS) [25]. Consistently, the expression of ascorbate peroxidase is up-regulated and also confirmed by qPCR (Additional file 1: Table S4, Additional file 3).

\section{Starch and sucrose metabolism}

Starch and sucrose metabolism is significantly enriched by KEGG Pathway analysis on up-regulated genes. On 0.5-h of stress, the expression of starch phosphorylase (PYG, 2.4.1.1), which catalyzes the hydrolysis of starch into alpha-D-glucose 1-phosphate, is significantly upregulated (Additional file 1: Table S4). At the same time, the expression of phosphoglucomutase (PGM, 5.4.2.2, catalyzing alpha-D-glucose 1-phosphate to alpha-D-glucose 6phosphate) and glucose-6-phosphate isomerase (GPI, 5.3.1.9, catalyzing alpha-D-glucose 6-phosphate to beta-Dfructose-6-phosphate) are significantly up-regulated (Additional file 1: Table S4), implying the alpha-D-glucose 1-phosphate from starch hydrolysis may go into glycolysis pathway (Fig. 3). On 1-h of stress, beta-fructofuranosidase (3.2.1.26, not shown on Fig. 3) and beta-amylase (3.2.1.2) are significantly up-regulated. On 2-h of stress, alphaamylase (3.2.1.1), trehalose 6-phosphate synthase (otsA, 2.4.1.15) and trehalose 6-phosphate phosphatase (otsB, 3.1.3.12) are significantly up-regulated (Fig. 3).

On the whole, genes catalyzing the hydrolysis of polysaccharide (such as starch and maltodextrin) and disaccharide (such as sucrose and maltose) are significantly up-regulated (Table 3). Other up-regulated genes besides polysaccharide hydrolysis, include trehalose 6-phosphate synthase (otsA, 2.4.1.15) and trehalose 6-phosphate phosphatase (otsB, 3.1.3.12) (Table 3). The up-regulation of otsA and otsB synchronously indicates the accumulating of trehalose (Fig. 3), which is not a reducing sugar and reported to play a role in abiotic stress tolerance [26]. The existing of PYG, alpha-amylase, beta-amylase, isoamylase (ISA, 3.2.1.68), and cyclomaltodextrin glucanotransferase (cgt, EC: 2.4.1.19, not shown on Fig. 3) indicates that there are alternative pathways for starch hydrolysis.

\section{Glycolysis and glycerol synthesis}

Glycolysis is significantly enriched by KEGG Pathway analysis on up-regulated genes. The up-regulations of PGM, GPI, the rate-limiting enzyme PFK1 (6-phosphofructokinase 1, 2.7.1.11), and fructose-bisphosphate aldolase were seen on 0.5 -h of stress (Additional file 1: Table S4), which implies alphpa-D-Glucose-1p from hydrolysis of starch goes to glycolysis (Fig. 4). Interestingly, 


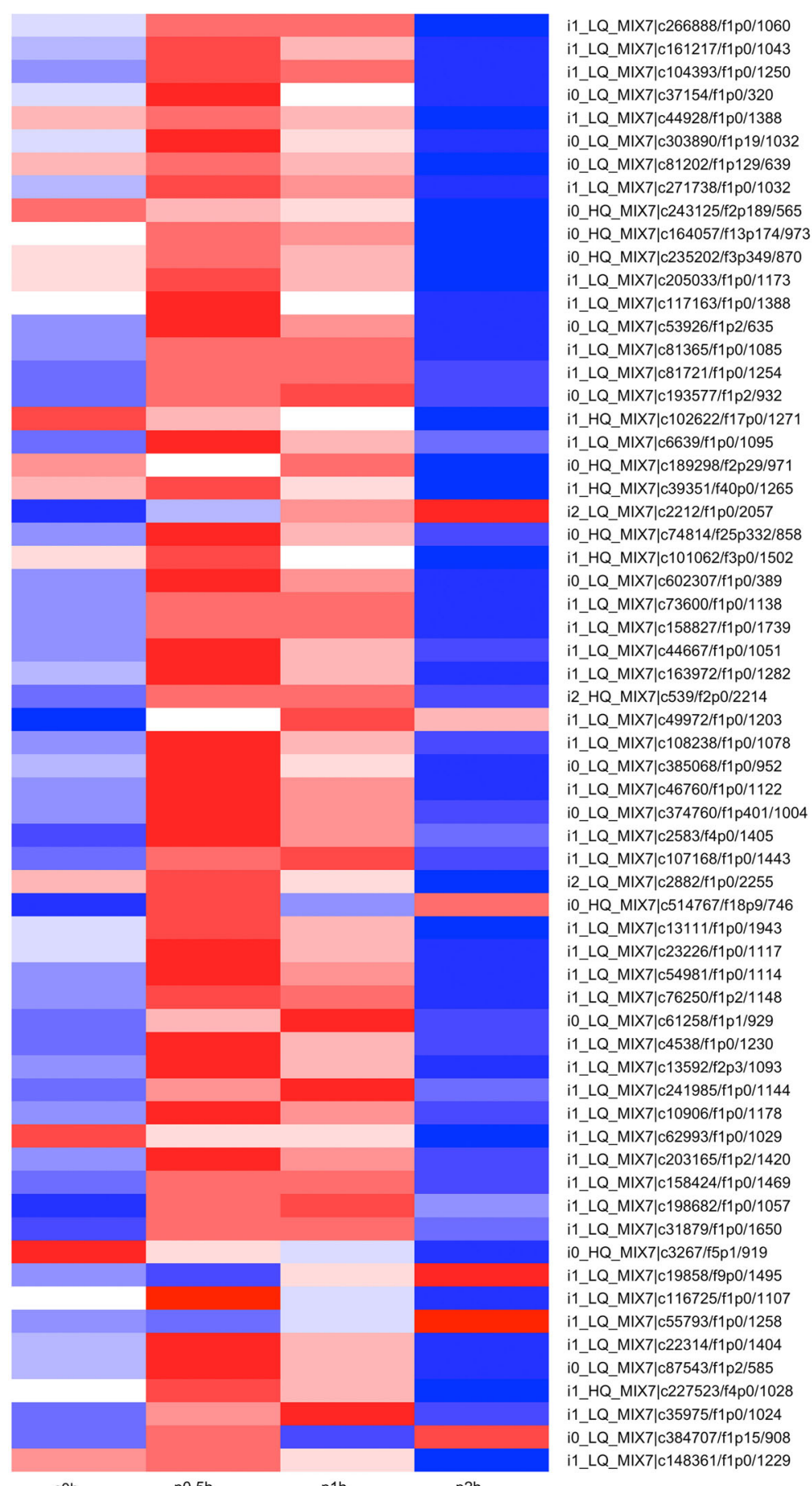

Fig. 2 Heat-map of photosynthesis; the colors from blue to red represent the gene express values from low to high. The z-scores represent gene expression values were generated from their FPKMs. The four columns represent the four experimental groups. COh represents the control group with no hypersaline stress applied. p0.5h, p1 h, and p2 h represent the three hypersaline treated groups with 0.5-h, 1-h, and 2-h time duration. Genes IDs are on the right. Genes are also grouped base on their expression patterns 


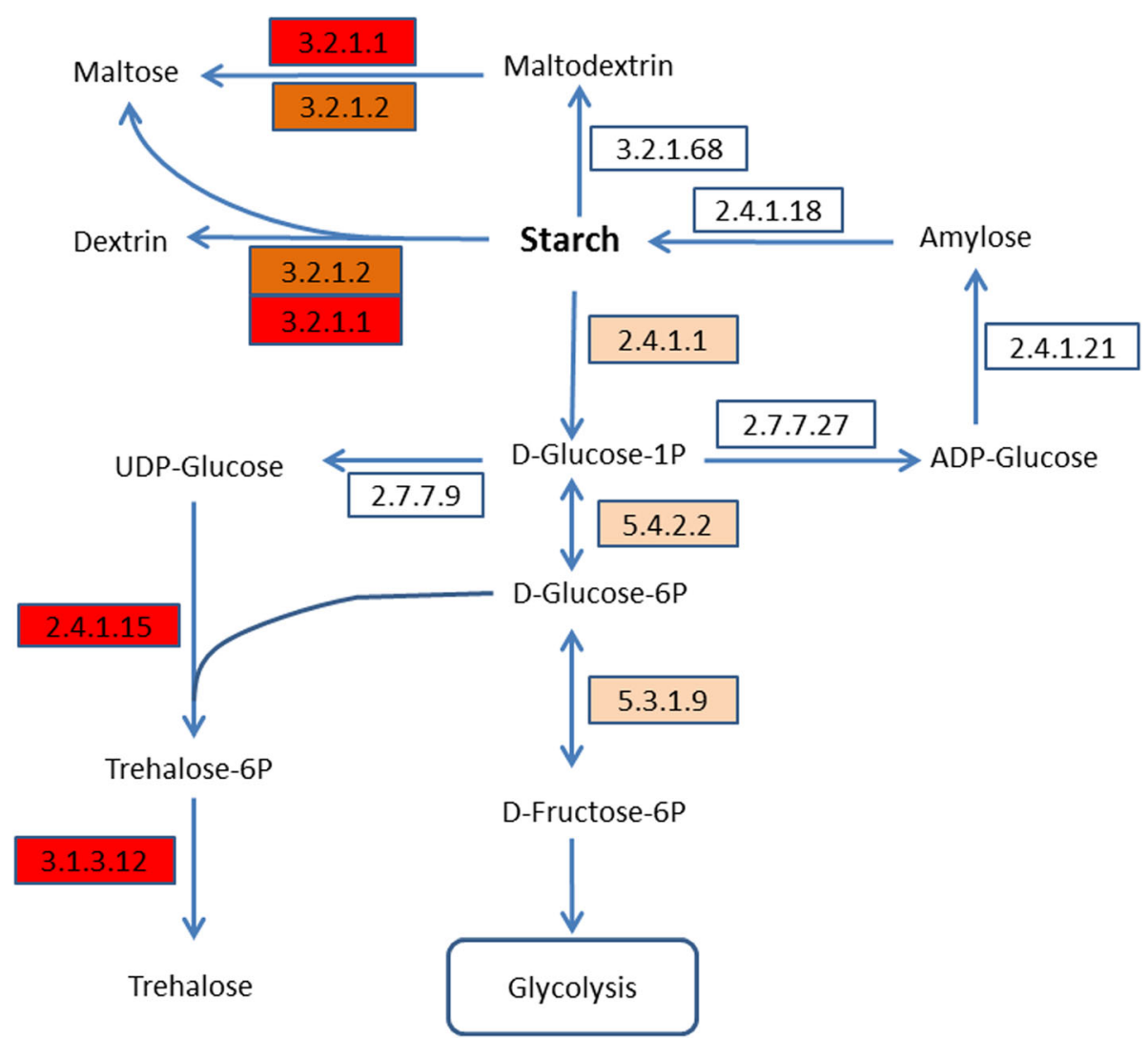

Fig. 3 The simplified pathway of starch metabolism. The numbers in the rectangles are enzyme codes, all the enzymes are identified in the transcriptome, the arrows show the direction of enzyme-catalyzed reaction; enzymes up-regulated on 0.5-h of stress are highlighted by light orange; enzymes up-regulated on 1-h of stress are highlighted by orange, enzymes up-regulated on 0.5-h of stress were also up-regulated on 1-h of stress; enzymes up-regulated on 2-h of stress are highlighted by red, enzymes up-regulated on 0.5-h and 1-h of stress were also up-regulated on 2 -h of stress

triosephosphate isomerase (TPI, 5.3.1.1), which catalyzing the reversible interconversion of Glyceraldehyde 3-phosphate (GADP) and Glycerone phosphate (also known as Dihydroxyacetone phosphate, DHAP), was significantly up-regulated on 0.5 -h of stress.
Our data show that the Dunaliella salina specific didomain glycerol-3-phosphate dehydrogenase (DsGPDH) can convert DHAP (an intermediate of glycolysis) to glycerol directly [27]. So the glycerol synthesis pathway of Dunaliella salina can be drawn based on the genes from

Table 3 Up-regulated enzymes involved in starch and sucrose metabolism

\begin{tabular}{|c|c|c|}
\hline Enzyme code & Name & Reaction \\
\hline \multicolumn{3}{|c|}{ Polysaccharide degradation } \\
\hline 2.4.1.1 & Glycogen phosphorylase & $\begin{array}{l}{[(1->4) \text {-alpha-D-glucosyl] } n+\text { phosphate }=[(1->4) \text {-alpha-D-glucosyl] } n-1} \\
+ \text { alpha-D-glucose 1-phosphate }\end{array}$ \\
\hline 3.2.1.1 & alpha-amylase & Starch $+\mathrm{H} 2 \mathrm{O}<=>$ Dextrin + Starch \\
\hline 3.2.1.2 & beta-amylase & Starch $<=>$ Dextrin + Maltose \\
\hline \multicolumn{3}{|c|}{ Disaccharide degradation } \\
\hline 3.2.1.26 & beta-fructofuranosidase & Sucrose $+\mathrm{H} 2 \mathrm{O}<=>$ D-Fructose + D-Glucose \\
\hline 2.4.1.25 & 4-alpha-glucanotransferase & Amylose $+\mathrm{n}$ D-Glucose $<=>$ n Maltose \\
\hline \multicolumn{3}{|l|}{ Others } \\
\hline 2.4.1.15 & trehalose 6-phosphate synthase & UDP-glucose + D-Glucose 6-phosphate $<=>$ UDP + alpha,alpha'-Trehalose 6-phosphate \\
\hline 3.1.3.12 & trehalose 6-phosphate phosphatase & alpha,alpha'-Trehalose 6-phosphate $+\mathrm{H} 2 \mathrm{O}<=>$ alpha,alpha-Trehalose + Orthophosphate \\
\hline
\end{tabular}




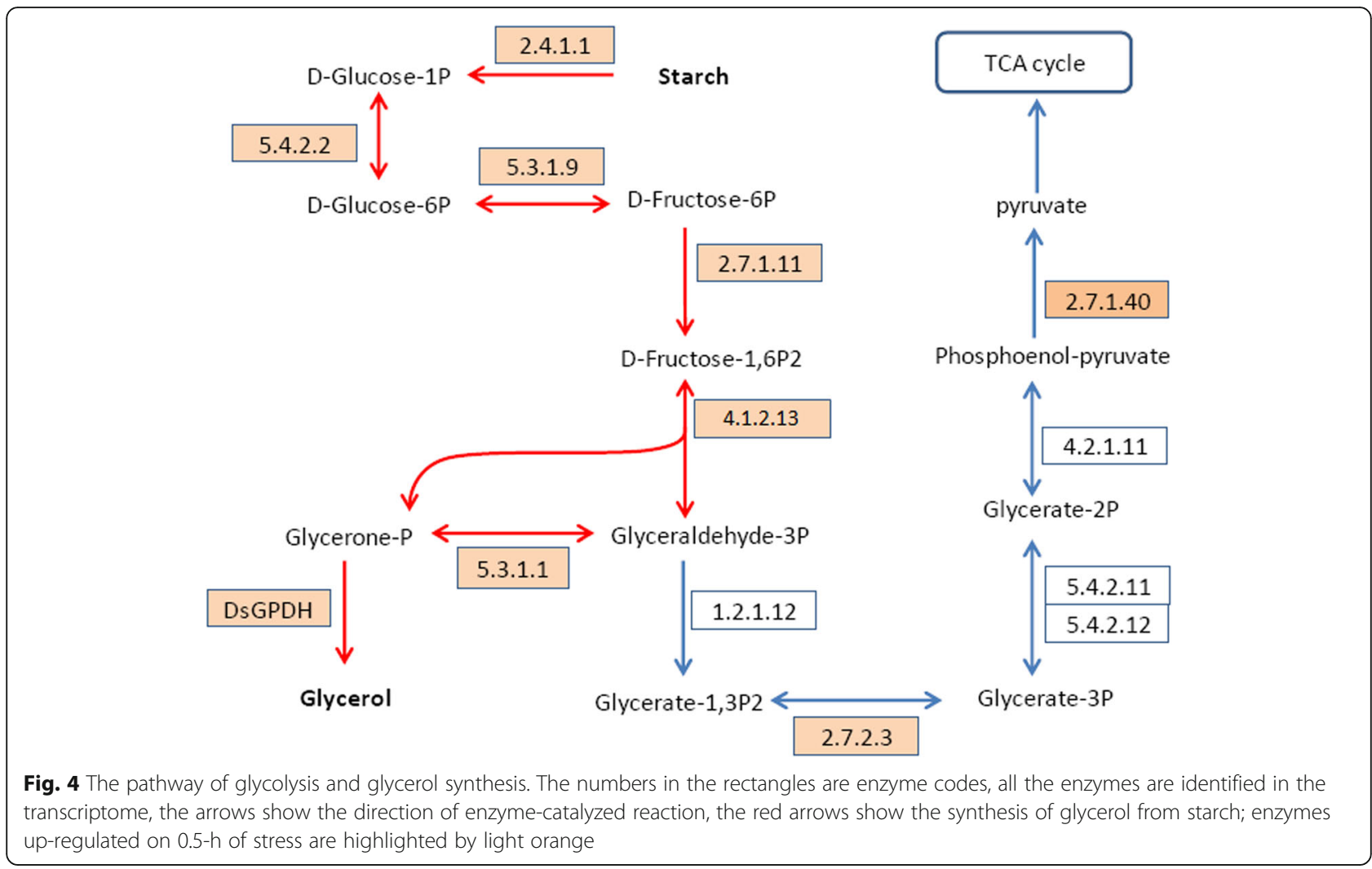

the transcriptomic data (Fig. 4). The new glycerol synthesis pathway includes only 6 enzyme-catalyzing steps to convert starch to glycerol, the six enzymes are all up-regulated on 0.5 -h of stress. The up-regulation of TPI can be explained as that more enzyme is needed to converting GADP to DHAP for glycerol synthesis. Consistently, DsGPDH is up-regulated 0.5, 0.9 and 1.0 fold on $0.5-\mathrm{h}, 1-\mathrm{h}$, and $2-\mathrm{h}$ of stress respectively (Additional file 1: Table S4).

\section{Lipid metabolism}

On 0.5 -h of stress, fatty acid biosynthesis is significantly enriched by KEGG Pathway analysis on up-regulated genes. The number of genes involved in this pathway remains stable on 1-h of stress, and decreases a little on 2$\mathrm{h}$ of stress. While the gene number of fatty acid degradation and biosynthesis of unsaturated fatty acids increase constantly (Table 4), which implies that fatty acid biosynthesis and degradation occurs synchronously when salt stress is applied. The up-regulated genes of fatty acid biosynthesis include acetyl-CoA carboxylase, long-chain acyl-CoA synthetase, FabZ (3-hydroxyacyl-[acyl-carrierprotein] dehydratase), enoyl-[acyl-carrier-protein] reductase, oleoyl-acyl carrier protein thioesterase, acyl-[acylcarrier-protein] desaturase, and biotin carboxyl carrier protein of acetyl-CoA carboxylase. However the upregulation of acetyl-CoA carboxylase wasn't confirmed by qPCR, but the up-regulation of acetyl-CoA carboxylase beta subunit was confirmed by qPCR (Additional file 3). On 2-h of stress, key genes involved in biosynthesis of unsaturated fatty acids are also significantly up-regulated, including delta- 7 desaturase, omega- 3 fatty acid desaturase and delta-12 desaturase (omega- 6 fatty acid desaturase), acyl-[acyl-carrier-protein] desaturase and acyl-CoA oxidase, implying the synthesis of unsaturated fatty acids (Additional file 2: Figure S4). The genes classified into fatty acid degradation by KEGG include long-chain acylCoA synthetase, acyl-CoA oxidase, alcohol dehydrogenase class-3, acetyl-CoA c-acetyltransferase, and glyoxysomal fatty acid beta-oxidation multifunctional protein MFP-a. Among them, long-chain acyl-CoA synthetase and acylCoA oxidase are also classified into fatty acid biosynthesis, but the up-regulation of the latter three genes implies the degradation of fatty acid.

On the other hand, the number of up-regulated genes involved in Glycerophospholipid and glycerolipid metabolism increase constantly with the increasing of stress time (Table 4). Genes catalyzing the formation of phospatidylcholine (lecithin), phosphatidylethanolamine, phosphatidyl-L-serine, phosphatidylglycerol, and phosphatidyl-D-myo-inositol are significantly up-regulated on 2-h of stress (Additional file 1: Table S4), indicating the synthesis of glycerophospholipid on 2-h of stress. Study reported that fatty acids of Dunaliella salina plasma 
Table 4 Fatty acid and lipid metabolism related terms enriched by KEGG Pathway

\begin{tabular}{|c|c|c|c|c|}
\hline & & \multicolumn{3}{|l|}{ samples } \\
\hline & & $0.5 \mathrm{~h}$ & $1 \mathrm{~h}$ & $2 \mathrm{~h}$ \\
\hline \multicolumn{5}{|l|}{ (Fatty acid) } \\
\hline KEGG_ID & KEGG_term & Number of Genes involved & Number of Genes involved & Number of Genes involved \\
\hline ko00061 & Fatty acid biosynthesis & $7^{\mathrm{a}}$ & $7^{\mathrm{a}}$ & 5 \\
\hline ko00071 & Fatty acid degradation & 1 & 2 & $7^{\mathrm{a}}$ \\
\hline ko01040 & Biosynthesis of unsaturated fatty acids & 1 & 2 & 5 \\
\hline \multicolumn{5}{|l|}{ (Lipid) } \\
\hline ko00564 & Glycerophospholipid metabolism & 1 & $8^{\mathrm{a}}$ & $14^{a}$ \\
\hline ko00561 & Glycerolipid metabolism & 0 & 4 & $10^{\mathrm{a}}$ \\
\hline \multicolumn{5}{|l|}{ (other) } \\
\hline ko04144 & endocytosis & 3 & 8 & $24^{\mathrm{a}}$ \\
\hline
\end{tabular}

${ }_{\text {indicates significantly enriched }}$

membrane and microsomal undergo changes in desaturation pattern under salt stress. They hypothesized that unsaturated fatty acids could help to keep fluidity of membrane when confronting salt stress [28]. So it is possible that the new synthesized unsaturated fatty acids might be incorporated into the new glycerophospholipids.

Interestingly, endocytosis, which can absorb and start the degradation of cell membrane [29], is significantly enriched on 2-h of stress (Table 4). When confronting salt stress, Dunaliella cells begin to shrink to cause excessive cell membrane. It is possible that the excessive membrane is degraded into building blocks for synthesis of new glycerophospholipid with unsaturated fatty acids, or further degraded for fuel energy metabolism.

\section{Amino acids and protein metabolism}

Several amino acid metabolism pathways are enriched by KEGG Pathway analysis on up-regulated genes (Table 5), including some high abundant amino acids such as glutamine, glutamic acid, serine, alanine, proline, aspartic acid and asparagine. These high abundant amino acids were reported to be synthesized during abiotic stress to act as compatible osmolytes, precursors for secondary metabolites, or storage forms of organic nitrogen [30]. Several amino acid biosynthesis pathways are also enriched especially on 2-h of stress, including phenylalanine, tyrosine and tryptophan biosynthesis, lysine biosynthesis, valine, leucine and isoleucine biosynthesis, and histidine metabolism (Table 5). These are low abundant amino acids and the synthesis of these amino acids is energetically costly and requires multiple reaction steps [30]. With the stress time increasing, the number of genes increased in all the pathways except arginine biosynthesis which remains stable, the increasing is very slowly or stable from 0.5 -h of stress to 1 -h of stress and sharply from 1-h of stress to 2-h of stress (Table 5). The sharp increase of gene numbers implies that the synthesis of these low abundant amino acids at a significantly higher rate on 2 -h of stress. Ribosome biogenesis and transcription are also significantly

Table 5 Amino acid metabolism related terms enriched by KEGG Pathway

\begin{tabular}{|c|c|c|c|c|}
\hline \multirow[t]{3}{*}{ KO_Term } & \multirow[t]{3}{*}{ KO_ID } & \multicolumn{3}{|l|}{ samples } \\
\hline & & $0.5 \mathrm{~h}$ & $1 \mathrm{~h}$ & $2 \mathrm{~h}$ \\
\hline & & Number of Genes involved & Number of Genes involved & Number of Genes involved \\
\hline Glycine, serine and threonine metabolism & ko00260 & $11^{\mathrm{a}}$ & $15^{\mathrm{a}}$ & $22^{\mathrm{a}}$ \\
\hline Alanine, aspartate and glutamate metabolism & ko00250 & 9 & 11 & 17 \\
\hline Arginine and proline metabolism & ko00330 & 2 & 5 & $11^{\mathrm{a}}$ \\
\hline Cysteine and methionine metabolism & ko00270 & $11^{\mathrm{a}}$ & 13 & $24^{\mathrm{a}}$ \\
\hline Phenylalanine, tyrosine and tryptophan biosynthesis & ko00400 & 4 & 4 & 14 \\
\hline Lysine biosynthesis & ko00300 & 1 & 4 & $9^{\mathrm{a}}$ \\
\hline Valine, leucine and isoleucine biosynthesis & ko00290 & 0 & 2 & $12^{\mathrm{a}}$ \\
\hline Histidine metabolism & ko00340 & 0 & 0 & 4 \\
\hline Arginine biosynthesis & ko00220 & 6 & 5 & 7 \\
\hline
\end{tabular}

indicates significantly enriched ( $P$ value $\leq 0.05)$ 
enriched on 2-h of stress (Table 6). Genes involved in the two biological processes are highly expressed on 2-h of stress (Additional file 2: Figure S5 and S6). These together implies the synthesis of new protein on 2-h of stress at a significantly higher rate, compared with that of $0.5-\mathrm{h}$ and $1-\mathrm{h}$ of stress. Spliceosome is significantly enriched on 1-h and 2-h of stress (Table 6; Additional file 2: Figure S7). Post-transcriptional regulation mediated by spliceosome is an important way of gene regulation and was reported to involve in responses to various abiotic stress including salt and temperature stress [31,32]. Alternative splicing is a fast way to generate new mRNAs (splice variants) using the existed pre-mRNAs, and then these splice variants can be translated into different proteins. Protein processing in endoplasmic reticulum is also significantly enriched on 1$\mathrm{h}$ and 2-h of stress (Table 6; Additional file 2: Figure S8), which implies the regulation at post-translational level.

On the other hand, ubiquitin mediated proteolysis is significantly enriched on 2-h of stress, which implies the degradation of proteins (Table 6; Additional file 2: Figure S9). The enrichments of protein synthesis and degradation at the same time implies a higher protein turnover rate on 2-h of stress. Some proteins are destined to degradation, such as misfolded proteins caused by stress [33], while new proteins are needed to adapt to the hypersaline environmental, such as chaperones (heat shock proteins) and reactive oxygen species scavenging enzymes (superoxide dismutase, catalase, and peroxiredoxins) [34]. Consistently, the expressions of these genes are up-regulated (Additional file 1: Table S4; Additional file 3).

\section{Ion homeostasis}

Surprisedly, the expression of all the possible $\mathrm{Na}^{+}$and $\mathrm{Cl}^{-}$transporters or channels are not up-regulated up to 2-h of stress (Table 7), which implies that the enhancement of exclusion of $\mathrm{Na}^{+}$or $\mathrm{Cl}^{-}$are not needed, probably because the extracellular ions can't go into Dunaliella cell easily. When confronting salt stress, Dunaliella cells undergo rapid shrinking by efflux of water through aquaporin [1]. The cell shrinkage and efflux of water result in the increased solutes concentrations in cytoplasm, and at the same time the glycerol synthesis starts, which contributes to the osmotic balance between cell membrane. From this point of view, if Dunaliella cells can restrict ion permission, the enhanced exclusion of the ions by membrane transporters seems no need, so we can't see the up-regulation of these ion transporters.

Some other metal ion transporters are also not upregulated on 0.5 - $\mathrm{h}$ and 1 -h of stress (Additional file 1: Table S5), while some metal ion transporters such as magnesium, zinc, and boron transporters are upregulated on 2-h of stress. It is possible that the upregulation of these transporters is to uptake metal ion for protein synthesis since Dunaliella cells began to synthesize large amounts of protein on 2-h of stress (Table 6).

\section{Salt stress sensing and signal transduction}

Kinetic of glycerol synthesis in Dunaliella shows that the synthesis can be detected within minutes and is independent of protein synthesis [35], which indicates that

Table 6 Protein metabolism related terms enriched by GO and KEGG Pathway

\begin{tabular}{|c|c|c|c|c|}
\hline & & \multicolumn{3}{|l|}{ samples } \\
\hline & & $0.5 \mathrm{~h}$ & $1 \mathrm{~h}$ & $2 \mathrm{~h}$ \\
\hline \multicolumn{5}{|l|}{ (transcription) } \\
\hline GO_ID & GO_term & Number of Genes involved & Number of Genes involved & Number of Genes involved \\
\hline GO:0006351 & transcription, DNA-templated & 26 & 56 & 191 \\
\hline GO:0006352 & DNA-templated transcription, initiation & 3 & 15 & $54^{\mathrm{a}}$ \\
\hline GO:0006355 & regulation of transcription, DNA-templated & 22 & 42 & 125 \\
\hline \multicolumn{5}{|l|}{ (translation) } \\
\hline GO:0006412 & translation & 50 & 51 & 127 \\
\hline GO:0006413 & translational initiation & 1 & 1 & 29 \\
\hline GO:0006417 & regulation of translation & 3 & 5 & 37 \\
\hline \multicolumn{5}{|l|}{ (other process) } \\
\hline $\mathrm{KO} \_\mathrm{ID}$ & KO_term & Number of Genes involved & Number of Genes involved & Number of Genes involved \\
\hline ko03008 & Ribosome biogenesis in eukaryotes & 0 & 1 & $33^{\mathrm{a}}$ \\
\hline ko03040 & Spliceosome & 9 & $20^{\mathrm{a}}$ & $74^{\mathrm{a}}$ \\
\hline ko04141 & Protein processing in endoplasmic reticulum & 11 & $30^{a}$ & $51^{a}$ \\
\hline ko04120 & Ubiquitin mediated proteolysis & 1 & 2 & $13^{\mathrm{a}}$ \\
\hline
\end{tabular}

${ }^{a}$ indicates significantly enriched ( $P$ value $\left.\leq 0.05\right)$ 
Table 7 The expressions of the possible sodium and chloride ion transporters or channels identified in the transcriptiome

\begin{tabular}{|c|c|c|c|c|c|}
\hline \multirow[t]{2}{*}{ Gene ID } & \multirow[t]{2}{*}{ Swissprot Description } & \multirow[t]{2}{*}{ PFAM description } & \multicolumn{3}{|c|}{$\log 2\left(\right.$ FoldChange) $^{a}$} \\
\hline & & & $0.5 \mathrm{~h}$ vs $0 \mathrm{~h}$ & $1 \mathrm{~h}$ vs $0 \mathrm{~h}$ & $2 \mathrm{~h}$ vs $0 \mathrm{~h}$ \\
\hline \multicolumn{6}{|l|}{ (Sodium channels) } \\
\hline i0_LQ_MIX7|c93492/f1 p0/580 & Sodium/sulfate cotransporter & - & FALSE & FALSE & FALSE \\
\hline i2_LQ_MIX7|c2141/f1p8/2443 & $\begin{array}{l}\text { Sodium-dependent phosphate } \\
\text { transporter }\end{array}$ & - & FALSE & FALSE & FALSE \\
\hline i1_LQ_MIX7|C73474/f1p0/1231 & sodium/metabolite cotransporter & - & FALSE & FALSE & FALSE \\
\hline i1_LQ_MIX7|c78858/f1p0/1342 & $\begin{array}{l}\text { Probable sodium/sulfate } \\
\text { cotransporter }\end{array}$ & - & FALSE & FALSE & FALSE \\
\hline i1_HQ_MIX7|c19950/f2p0/1494 & Sodium channel protein $60 \mathrm{E}$ & - & FALSE & FALSE & FALSE \\
\hline i1_LQ_MIX7|c50163/f1p0/1376 & sodium/metabolite cotransporter & - & FALSE & FALSE & FALSE \\
\hline i1_LQ_MIX7|c105505/f1 p0/1621 & sodium/metabolite cotransporter & - & FALSE & FALSE & FALSE \\
\hline i0_LQ_MIX7|c223101/f1p0/398 & Sodium/calcium exchanger & - & FALSE & FALSE & FALSE \\
\hline i2_LQ_MIX7|c1504/f1 p0/2682 & Urea-proton symporter & - & FALSE & DOWN & DOWN \\
\hline i1_LQ_MIX7|c78130/f1p0/1314 & - & Sodium/calcium exchanger protein & FALSE & FALSE & FALSE \\
\hline iO_LQ_MIX7|C11594/f1p1/871 & - & Sodium / potassium ATPase beta chain & FALSE & FALSE & FALSE \\
\hline i0_LQ_MIX7|c172741/f1p0/649 & - & $\begin{array}{l}\text { Sodium/glutamate symporter//Ureide } \\
\text { permease }\end{array}$ & FALSE & FALSE & FALSE \\
\hline i0_LQ_MIX7|c4443/f1p1/741 & - & Amiloride-sensitive sodium channel & FALSE & FALSE & FALSE \\
\hline i1_LQ_MIX7|c29086/f1p0/1191 & - & Sodium/calcium exchanger protein & FALSE & FALSE & FALSE \\
\hline i1_LQ_MIX7|c27958/f1p0/1854 & - & $\begin{array}{l}\text { Sodium ion transport-associated//Rer1 } \\
\text { family }\end{array}$ & FALSE & FALSE & FALSE \\
\hline i0_LQ_MIX7|c5202/f1p0/598 & - & Sodium:neurotransmitter symporter family & FALSE & FALSE & DOWN \\
\hline i1_LQ_MIX7|C13817/f1p0/1659 & - & $\begin{array}{l}\text { Amiloride-sensitive sodium channel//EGF- } \\
\text { like domain }\end{array}$ & FALSE & FALSE & FALSE \\
\hline iO_HQ_MIX7|c12934/f2p1/896 & - & $\mathrm{Na}+/ \mathrm{H}+$ ion antiporter subunit & FALSE & FALSE & FALSE \\
\hline \multicolumn{6}{|l|}{ (chloride channels) } \\
\hline i1_LQ_MIX7|c29481/f1p0/1076 & Chloride channel protein CLC-f & - & FALSE & FALSE & FALSE \\
\hline i2_LQ_MIX7|c3324/f1 p0/2034 & Chloride channel protein CLC-f & - & FALSE & FALSE & FALSE \\
\hline i1_LQ_MIX7|c13569/f1p0/1372 & - & $\begin{array}{l}\text { Dimerisation domain of } \mathrm{Ca}+- \text {-activated } \\
\text { chloride-channel, anoctamin }\end{array}$ & FALSE & FALSE & FALSE \\
\hline
\end{tabular}

a"FALSE" means no significant up- or down-regulation, "DOWN" means significant down-regulation

the expression of the signal receptors and signal transducers might not be changed upon signal stimulation. Therefore, using the GO enrichments of the up-regulated genes to show the involved signaling pathways may not reflect the real truth. So far, very little has been known about the sensing and signal transduction cascades of salt stress in Dunaliella. For this reason, we tried to search the possible cell surface receptors based on the sequence annotation data. Two possible receptors are found: a putative Gprotien coupled seven-transmembrane receptor (GPCR) and a receptor-like serine/threonine protein kinase. Small GTP-binding proteins were identified in D. salina plasma membrane by Mass Spectrometry and were thought to involve in sensing and signaling of salt stress in $D$. salina [8]. However, the ligands of the two receptors are less likely to be $\mathrm{Na}^{+}$or $\mathrm{Cl}^{-}$. Due to the properties of salt stress, we focused on ion channels. Genes encoding sodium channel, chloride channel and mechanosensitive ion channel are found in the sequence data.

One gene is annotated as a sodium channel, which shows sequence similarity with Sodium channel protein 60E from Drosophila melanogaster. Sodium channel protein $60 \mathrm{E}$ is a voltage-gated sodium channel, and the opening and closing of which is triggered by changing the ion concentration, and hence the charge gradient, between the two sides of the cell membrane [36]. In rabbit chemoreceptor cells, the activation of voltagegated sodium channels can cause cell membrane depolarization, which will result in $\mathrm{Ca}^{2+}$ influx by activation of voltage-dependent $\mathrm{Ca}^{2+}$ channels, so the signal is switched to intracellular $\mathrm{Ca}^{2+}$ signal $[37,38]$. No sequences encoding voltage-dependent $\mathrm{Ca}^{2+}$ channel are found in the sequence data. It cannot be concluded that there are no voltage-dependent $\mathrm{Ca}^{2+}$ channels in 
Dunaliella salina since about $40 \%$ of the transcriptome cannot be annotated by the present database. Instead, sequences encoding sodium/calcium exchanger, $\mathrm{Ca}^{2+}$ transporting ATPase and calcium uniporter are found in the sequence data. Furthermore, sequences encoding Calcium dependent kinases, $\mathrm{Ca}^{2+} /$ calmodulin-dependent protein phosphatase, Calcium sensing receptor, calmodulin, Calcium/calmodulin-dependent 3', 5'-cyclic nucleotide phosphodiesterase are also found in the sequence data. These indicate that $\mathrm{Ca}^{2+}$ plays an important role in signal transduction.

Two genes are annotated as voltage gated chloride channel. Since the salt stress was applied by only increasing the concentration of $\mathrm{NaCl}$ in medium, we wonder if the chloride channel can sense the salt stress by the influx of $\mathrm{Cl}^{-}$. WNK kinase (serine/threonine-protein kinase) is reported to function as a direct chloride sensor, and several genes encoding WNK are also found in the transcriptome. WNK are activated by reduction in intracellular $\mathrm{Cl}^{-}$concentrations and inactivated by binding of a $\mathrm{Cl}^{-}$. Chloride binding inhibited the autophosphorylation of WNK1, thereby inhibiting kinase activity $[39,40]$. WNK is also reported to inhibit sodium [41, 42] and potassium channel [43, 44]. Except for these, how the influx of $\mathrm{Cl}^{-}$triggers intracellular pathways are almost unknown.

Mechanosensitive ion channels will respond to the mechanical deformation, which includes changes in the tension, thickness, or curvature, of the membrane. Mechanosensitive channels respond to membrane tension by altering their conformation between an open state and a closed state $[45,46]$. When confronted with salt stress, Dunaliella cells shrink rapidly, thus the membrane deformation could cause the opening of mechanosensitive ion channels. One gene showing significant sequence similarity with the mechanosensitive ion channel protein 5 of Arabidopsis thaliana is found; however we don't know whether it is a calcium channel or other ion channel. The applying of salt stress can trigger the voltage-gated sodium channel, the voltage-gated chloride channel and also the mechanosensitive ion channel, it is hard to tell which one is the very first signal receptor. Dunaliella cells undergo rapid shrinkage by efflux of water through aquaporin [1]. So the mechanosensitive ion channel seems unlikely to be the first receptor, but might be the enhancer.

A previous paper showing that MAPK signaling cascade may be involved in the salt stress of Dunaliella by western blot analysis [47]. Many genes on MAPK signaling cascade are also found in the sequence data, including mitogen-activated protein kinase (MAPK), Mitogenactivated protein kinase kinase (MAP $2 \mathrm{~K}$ ), Mitogenactivated protein kinase kinase kinase (MAP $3 \mathrm{~K}$ ), Mitogen-activated protein kinase kinase kinase kinase
(MAP 4K), MAP kinase phosphatase 1, MAP kinase phosphatase 5, MAP kinase phosphatase 6, and dual specificity MAP kinase phosphatase, which indicates that MAPK signaling pathway may be involved in salt stress signal transduction of Dunaliella salina.

\section{Discussion}

Most of the qPCR results shew up-regulations on at least one of the three time points of hypersaline stress, which is consistent with the NGS results except catalase and acetyl-CoA carboxylase. No up-regulations of catalase and acetyl-CoA carboxylase were detected by $\mathrm{qPCR}$ (Additional file 3). Acetyl-CoA carboxylase, which converts cytosol acetyl-CoA into malonyl CoA, the first committed step in the synthesis of fatty acids, is a multisubunit enzyme in the chloroplasts of most plants and algae [48]. The up-regulation of acetyl-CoA carboxylase beta subunit was detected by qPCR. The up-regulation of its beta subunit could possibly imply the enhancement of the holoenzyme activity.

Dunaliella is a good model organism for studying salt tolerance. Omics methods are particularly appropriate to analyze the stress response of Dunaliella at a global level, because Dunaliella is a unicellular alga and there will be no problem caused by different cell types. Previous omics studies can't give a global understanding of the response process of Dunaliella to salt stress due to limited discovery of genes involved or unappropriated time duration of stress $[13,14]$. Here we reported the transcriptomic profiles on $0.5-\mathrm{h}, 1-\mathrm{h}$ and 2 -h of hypersaline stress and compared the profiles with that before stress. Our results imply that the response of Dunaliella to hypersaline stress is almost finished on 2-h stress. The reasons are that nuclear origin of replication recognition complex and reproductive process are significantly enriched on 2-h stress, which implies that the algae cell starts to reproduce on 2-h of stress and cells already adapted the new environment. This is in agreement with the previous studies reporting that intracellular glycerol synthesis accomplished and cells recovered their original volume in about $2 \mathrm{~h}$ after hypersaline stress $[15,16]$.

When the hypersaline stress is applied, many of the genes classified into photosynthesis show a pulse expression pattern (quick increasing and then quick decreasing), implies that hypersaline stress induces enhancement of photosynthesis, but no constant enhancement of photosynthesis is observed. Carbon fixation from photosynthesis has its upper limit under a given condition. Study reported that only a small part of carbon source of glycerol synthesis comes from $\mathrm{CO}_{2}$ fixation of photosynthesis, and others are from starch breakdown [49]. However, constant increasing of carbon fixation is observed, this may be induced by $\mathrm{NaHCO}_{3}$ in the high salt medium when stress is 
applied. Constant increase of pigment biosynthetic process including heme biosynthetic process is also observed. Pigments such as carotenoids play a role in response to abiotic stress [50], and heme could be used to synthesize heme-containing enzymes, such as catalase and ascorbate peroxidase, which play important roles in detoxification of reactive oxygen species (ROS) [25]. Starch is the main carbon source of glycerol synthesis when hypersaline stress is applied [50]. Consistently, starch and sucrose metabolism is significantly enriched. The new drawn glycerol synthesis pathway includes only 6 enzyme-catalyzing steps to convert starch to glycerol, which is theoretically faster than previous reported pathways $[9,51]$. Synthesis of unsaturated fatty acid is consistent with previous report [28]. Interestingly, endocytosis is significantly enriched, together with the enrichment of Glycerophospholipid metabolism. We speculate that endocytosis could cause the degradation of excessive plasma membrane and help the synthesis of new glycerophospholipid with unsaturated fatty acids.

From amino acids and protein metabolism, we found an indication of sharp increasing of protein synthesis rate on 2-h stress based on the significant enrichment of ribosome biogenesis and transcription. Enhancement of regulation on protein synthesis is also implied by the significant enrichment of spliceosome and protein processing in endoplasmic reticulum. While, the significant enrichment of ubiquitin mediated proteolysis implies the degradation of proteins. Taken together, accelerated protein turnover rate on 2-h of stress is implied.

It is very interesting that no sodium and chloride channels or transporters are up-regulated during the period of 2-h of stress. No up-regulation of $\mathrm{Na}^{+} / \mathrm{H}^{+}$antiporter was detected by qPCR (Additional file 3). This is in consistent with the reports of $\mathrm{Na}^{+} / \mathrm{H}^{+}$antiporter, which regards $\mathrm{Na}^{+} / \mathrm{H}^{+}$antiporter as the main player to eliminate $\mathrm{Na}^{+}$during salt stress $[7,52]$. We can't see the up-regulation of $\mathrm{Na}^{+} / \mathrm{H}^{+}$antiporter, maybe the activity of $\mathrm{Na}^{+} / \mathrm{H}^{+}$antiporter is regulated at protein level instead of mRNA level. However, if Dunaliella cell can restrict $\mathrm{Na}^{+}$permission, the exclusion of the ions by membrane transporters seems no need.

The time setting of stress gives us good results. We can see the constant increasing of the number of upregulated genes with the increasing of stress time. Many of the results of functional enrichment are consistent with the previous studies, such as the enhancement of photosynthesis and carbon fixation [9], the degradation of starch for glycerol synthesis [49], and the desaturation of membrane lipid [28] when hypersaline stress is applied.

We also have some new findings, such as the enhancement of heme biosynthetic process and endocytosis, the accelerated protein turnover rate, the enhancement of spliceosome and protein processing in endoplasmic reticulum in response to hypersaline stress. These new findings shed light on the mechanism of salt tolerance of photosynthetic plants and may help improving salt tolerance of crops by genetic manipulation.

Finally, we like to acknowledge the limitation of this study, that is, all the analyses are based on the transcriptomic data, the changes at mRNA level. Although protein levels are usually positively correlated with the mRNA levels, there are post-transcriptional and translational regulations that may affect the correlation. From this point of view, it is necessary to further figure out how a new intracellular balance at the physiological levels achieved in Dunaliella salina to adapt to hypersaline environment.

\section{Conclusion}

At global transcriptomic level, the response of Dunaliella salina to hypersaline stress is systematic, possibly involving enhancement of photosynthesis, constant increasing of carbon fixation and heme biosynthetic process, degradation of starch, synthesis of glycerol, membrane lipid desaturation, accelerated protein turnover, enhancement of spliceosome, protein processing in endoplasmic reticulum, and endocytosis. The changes of these biological processes will help to understand the achieved new balance adapting to the hypersaline environment.

\section{Methods}

\section{Algae and culture conditions}

D. salina strain CCAP 19/30 was obtained from Mariela A. González and Thomas Pröschold. The algae grew in a controlled-environment chamber at $20^{\circ} \mathrm{C}$ with $14 \mathrm{~h}$ lighting and $10 \mathrm{~h}$ darkness. The composition of the growth medium was $1 \mathrm{M} \mathrm{NaCl}, 5.0 \mathrm{mM} \mathrm{NaNO}_{3}, 5.0 \mathrm{mM}$ $\mathrm{MgSO}_{4 \_} \mathrm{HH}_{2} \mathrm{O}, 0.1 \mathrm{mM} \mathrm{NaH} \mathrm{PO}_{4 \_} 2 \mathrm{H}_{2} \mathrm{O}, 1.0 \mathrm{mM} \mathrm{KCl}$, $10.0 \mathrm{mM} \mathrm{NaHCO} 3,0.3 \mathrm{mM} \mathrm{CaCl}{ }_{2} 2 \mathrm{H}_{2} \mathrm{O}$ and a mixture of micronutrients [53]. Total $1200 \mathrm{~mL}$ algae in log phase (about $10^{6}$ cells $/ \mathrm{mL}$ ) were divided into 12 bottles, 100 $\mathrm{mL}$ per bottle. Three bottles were used for control; the other 9 bottles were used for hyper-salt stress treatment. For hyper-salt stress treatment, each of the 9 bottles was added directly with $100 \mathrm{~mL}$ of high salt medium (containing $4 \mathrm{M} \mathrm{NaCl}$ ) to form a final $\mathrm{NaCl}$ concentration of $2.5 \mathrm{M}$ and was further cultured in light at $20^{\circ} \mathrm{C}$. Algae cells under stress were harvested on time of $0.5-\mathrm{h}, 1-\mathrm{h}$, and 2-h for total RNA extraction. The control algae cells were also harvested for total RNA extraction. In all, there are 4 time points ( 4 groups), $0 \mathrm{~h}, 0.5 \mathrm{~h}, 1 \mathrm{~h}$ and $2 \mathrm{~h}$, each time point with three replicates. In total 12 RNA samples were prepared from 12 algae samples. 


\section{RNA extraction}

Total RNA was extracted with TRIzol (Invitrogen, USA) by following the user manual. RNA degradation and contamination was monitored on 1\% agarose gels. RNA purity and integrity was checked by NanoPhotometer (IMPLEN, Germany) and RNA Nano 6000 Assay Kit of the Bioanalyzer 2100 system (Agilent Technologies, USA). RNA concentration was measured by Qubit ${ }^{\bullet} 2.0$ Flurometer together with Qubit ${ }^{\circ}$ RNA Assay Kit (Life Technologies, USA).

\section{Hiseq libraries construction for the second generation sequencing}

The 12 RNA samples were used to construct 12 Hiseq libraries, one library per RNA sample. Three microgram total RNA per RNA sample was used to prepare the sequencing library with NEBNext Ultra RNA Library Prep Kit for Illumina (NEB, USA) by following the manufacturer's instruction. Briefly, mRNA was purified by using poly- $T$ magnetic beads and was fragmented to perform first-strand cDNA by using M-MuLV reverse transcriptase and random hexamer-primers. Second-strand cDNA was synthesized by using DNA polymerase I and RNase $\mathrm{H}$. The cDNA was end-repaired and A-tailed, and NEBNext Adaptor with hairpin loop structure was linked. After size selection with AMPure XP system (Beckman Coulter, Beverly, USA) and PCR amplification with Phusion High-Fidelity DNA polymerase, samples were sequenced on the Illumina Hi-Seq 2000 system, generating paired-end (PE) reads with a length of $150 \mathrm{bp}$.

\section{PacBio library construction for the third generation sequencing}

The only reported Dunaliella salina (CCAP19/18) genome shows low sequence similarity with our algae strain (CCAP 19/30) and can't be used as the reference genome for mRNA sequence assembly and annotation, so the third generation sequencing was applied to generate the full length mRNA sequences. One microgram mRNA (generated by equally mixing mRNAs from the 9 stress samples and the 3 control samples) were used for first-strand cDNA synthesis with SMARTer PCR cDNA Synthesis Kit (Clontech Cat. 634,926). Double-strand cDNA was amplified with PrimeSTAR GXL DNA Polymerase (Clontech R050B). The $>4 \mathrm{~kb}$ cDNA fraction was generated with the BluePippin Size Selection System and mixed with the cDNA before fractionation at 1:1 ratio. The mixed cDNA was used to construct a combined SMRTbell Libray for sequencing. The library was subsequently sequenced on the PacBio Sequel System platform using SMRT Cell $1 \mathrm{M}$ v2.

\section{Data processing and transcriptome assembly}

Firstly, circular consensus sequences (CCS) were generated from Pacbio raw reads. Then, full length non chimera
(FLNC) reads were generated from CCS. FLNC reads should have sequence regarding to both $5^{\prime}$ and $3^{\prime}$ Clontech kit primers as well as a poly-A tail signal preceding the $3^{\prime}$ primer. FLNC reads were clustered by ICE (Iterative isoform-clustering) and a consensus sequence was generated from each cluster. The consensus sequences were corrected by non-full length sequences to generate the polished consensus sequences.

Hiseq raw reads were processed to generate Hiseq clean reads by removing adapter sequences, excluding reads contains $>50 \%$ nucleotides with Qphred $\leq 20$, and reads with ambiguous bases $\mathrm{N}$. Hiseq clean reads were used to correct the polished consensus sequences obtained from the third generation sequencing. The correction process was done by Proovread [54]. The final corrected unique full length sequences were used for annotation and gene expression analysis.

\section{Coding sequence analysis and gene annotation}

Transcripts were firstly submitted to $\mathrm{Nr}$ and Swissprot protein database to analyze the potential open reading frames (ORF), then the transcripts failed to hit any proteins in the two databases were submitted to the software ESTSCAN (3.0.3) [55] to analyze the potential ORF, finally the transcripts failed the ESTSCAN are defined as non-coding sequences. The corrected unique full length sequences were annotated against 7 databases, $\mathrm{Nr}$ (NCBI's non-redundant protein sequences), $\mathrm{Nt}$ (NCBI's nucleotide sequences), Pfam (protein family), KOG/COG (clusters of orthologous groups of proteins/ eukaryotic ortholog groups), Swiss-Prot, KEGG (Kyoto encyclopedia of genes and genomes) and GO (gene ontology) database. The softwares used are diamond v0.8.22 for $\mathrm{Nr}$ (e-value=1e-5), KOG/COG (1e-3) and Swiss-Prot (e-value =1e-5); NCBI blast 2.2.28 for Nt (evalue =1e-5); HMMER 3.0 for Pfam (e-value=0.01); KEGG automatic annotation server (KAAS) for KEGG (e-value $=1 \mathrm{e}-10)$, Blast2GO v2.5 for GO (e-value =1e-6). When available, the functional annotation from SwissProt was preferred because of its high accuracy.

\section{Quantification of gene expression level}

Briefly, Hiseq clean reads from each replicate were mapped to the corrected unique full-length sequences using HTSeq v0.6.1 [56]. And then FPKM (expected number of Fragments per Kilobase of transcript sequence per Millions base pairs sequenced) of each gene was calculated based on the length of the gene and reads count mapped to this gene. FPKM considers the effect of sequencing depth and gene length at the same time, and is currently the most commonly used method for estimating gene expression levels [57]. Clustering analysis of gene expression value was performed by SOM method [58]. 


\section{Differential expression assessment}

Differential expression analysis of two groups was performed by using the DESeq $\mathrm{R}$ package with the read counts from HTSeq [59]. DESeq provides statistical routines for determining differential expression in digital gene expression data, using a model based on the negative binomial distribution. The resulting $P$ values were adjusted using the Benjamini and Hochberg's approach for controlling the false discovery rate. Genes with an adjusted $P$-value $<0.05$ found by DESeq were assigned as differentially expressed.

\section{Gene enrichment analysis}

Gene Ontology (GO) terms were assigned to each genes based on the BLAST2GO [60]. Gene Ontology (GO) enrichment analysis of differentially expressed genes were implemented by the GOseq $R$ package [61], in which gene length bias was corrected. GO terms with corrected $P$ value $<0.05$ were considered significantly enriched by differential expressed genes.

KEGG pathways were assigned to each genes based on the KAAS. KOBAS software [62] was used to test the statistical enrichment of differential expression genes in KEGG pathways. The enrichment analysis was tested using a hypergeometric test at a significance cutoff of 0.05 false discovery rate (FDR). Pathways were assigned as significantly enriched if they had a FDR below $5 \%$.

\section{Real-time PCR (qPCR)}

In order to confirm the expression results from RNAsequencing, we perform qPCR analysis of the key genes from each functional group. The qPCR kit was from TaKaRa (DRR081A, TaKaRa Biotechnology, and Dalian, China). The primers used in qPCR were in the supplementary materials (Additional file 3 ). The reference gene used in qPCR was $18 \mathrm{~S}$ rRNA. The relative expression quantification was calculated by the $2^{-\Delta \Delta \mathrm{Ct}}$ method.

\section{Supplementary information}

Supplementary information accompanies this paper at https://doi.org/10. 1186/s12864-020-6507-2.

Additional file 1: Table S1. The data qualities of the libraries of the second-generation sequencing. Table S2. Transcripts properties from the third-generation sequencing. Table S3. GO terms enriched from the down-regulated genes. Table S4. Up-regulation of some key genes on different stress time compare with that before stress. Table S5. The expressions of the possible metal ion (excluding sodium and chloride ion) transporters or channels identified in the transcriptome.

Additional file 2: Figure S1. Clustering analysis of the differentially expressed genes in each sample. Figure S2. Heat-map of carbon fixation. Figure S3. heat-map of tetrapyrrole biosynthetic process. Figure S4. Heat-map of fatty acid biosynthesis. Figure S5. Heat-map of ribosome biogenesis. Figure $\mathbf{5 6}$. Heat-map of transcription. Figure S7. Heat-map of spliceosome. Figure S8. Heat-map of protein processing in endoplasmic reticulum. Figure $\mathbf{S 9}$. Heat-map of ubiquitin mediated proteolysis.
Additional file 3. Confirmation of the expressions of the key genes by qPCR.

\section{Abbreviations}

ALDO: Fructose-bisphosphate aldolase; CCAP: The Culture Collection of Algae and Protozoa; CCS: Circular consensus sequences; CEGs: Core eukaryotic genes; CGT: Cyclomaltodextrin glucanotransferase; DHAP: Dihydroxyacetone phosphate; DsGPDH: Dunaliella salina glycerol-3-phosphate dehydrogenase; EST: Expressed sequence tag; FDR: False discovery rate; FLNC: Full length non chimera; FPKM: Expected number of Fragments per Kilobase of transcript sequence per Millions base pairs sequenced; GADP: Glyceraldehyde 3phosphate; GO: Gene ontology; GPCR: G-protien coupled seventransmembrane receptor; GPDH: Glycerol-3-phosphate dehydrogenase; GPI: Glucose-6-phosphate isomerase; ICE: Iterative isoform-clustering; ISA: Isoamylase; KAAS: KEGG automatic annotation server; KEGG: Kyoto encyclopedia of genes and genomes; KOG/COG: Clusters of orthologous groups of proteins/eukaryotic ortholog groups; MAP: Mitogen-activated protein; MAPK: Mitogen-activated protein kinase; Nr: NCBl's non-redundant protein sequences; Nt: NCBI's nucleotide sequences; ORF: Open reading frames; OtsA: Trehalose 6-phosphate synthase; OtsB: Trehalose 6-phosphate phosphatase; Pfam: Protein family; PGM: Phosphoglucomutase; PYG: Starch phosphorylase; ROS: Reactive oxygen species; TPI: Triosephosphate isomerase; WNK kinase: With no lysine (K) kinase

\section{Acknowledgements}

We thank Dr. Min Yang and Dr. Jian-hua Tang for the language editing of this manuscript.

\section{Authors' contributions}

QZ directed the project; $\mathrm{QH}, \mathrm{YL}, \mathrm{HT}, \mathrm{YZ}, \mathrm{JG}$, and RL carried out the experiments; QH, YL, HT, YW, and QZ analyzed the data; $Q H, Q Z$ and $Y L$ wrote the manuscript with input from all members. All authors have read and approved the manuscript.

\section{Funding}

Funds were provided by Sichuan Province Health Department (17PJ512), Natural Science Foundation of China (81602653), the National Key Technology Support Program (2014BAD13B03), and the Fundamental Research Funds for the Central Universities, Southwest Minzu University (2017NZYQN36). The funders had no role in the study process including preparation of the manuscript.

\section{Availability of data and materials}

The datasets generated during the current study are deposited in the Sequence Read Archive under accession number SRR8552788, and SRR8543799 to SRR8543810. [https://trace.ncbi.nlm.nih.gov/Traces/sra/sra.cgi] The datasets will be available after the publishing of this manuscript or from the corresponding author on reasonable request.

Ethics approval and consent to participate Not applicable.

\section{Consent for publication}

Not applicable.

\section{Competing interests}

The authors declare that they have no competing interests.

\section{Author details}

${ }^{1}$ Key Laboratory of Qinghai-Tibetan Plateau Animal genetic Resource Reservation and Utilization, College of Life Science and Technology, Southwest Minzu University, Chengdu, People's Republic of China. ${ }^{2}$ Institute of Qinghai-Tibetan Plateau, Southwest Minzu University, Chengdu, People's Republic of China. ${ }^{3}$ Reproductive and endocrine laboratory, Chengdu Woman-Child Central Hospital, Chengdu 610051, People's Republic of China. ${ }^{4}$ School of Laboratory Medicine, Chengdu Medical College, Chengdu 610500, People's Republic of China. 
Received: 25 April 2019 Accepted: 20 January 2020

\section{Published online: 03 February 2020}

\section{References}

1. Ben-Amotz A, Avron M. The role of glycerol in the osmotic regulation of the Halophilic alga Dunaliella parva. Plant Physiol. 1973;51(5):875-8.

2. Avron M. The osmotic components of halotolerant algae. Trends Biochem Sci. 1986;11(1):5-6.

3. Wegmann K. Biochemische anpassung von Dunaliella an wechselnde salinität und temperatur. J Berichte der Deutschen Botanischen Gesellschaft. 1979;92(1):43-54.

4. Ben-Amotz A, Avron M. Isolation, characterization, and partial purification of a reduced Nicotinamide adenine dinucleotide phosphate-dependent Dihydroxyacetone Reductase from the Halophilic alga Dunaliella parva. Plant Physiol. 1974;53(4):628-31.

5. Gee R, Goyal A, Byerrum RU, Tolbert NE. Two isozymes of dihydroxyacetone phosphate reductase in Dunaliella. Plant Physiol. 1989;91(1):345-51.

6. Katz A, Kaback HR, Avron M. Na+/H+ antiport in isolated plasma membrane vesicles from the halotolerant alga Dunaliella salina. FEBS Lett. 1986;202(1): $141-4$.

7. Katz A, Kleyman TR, Pick U. Utilization of amiloride analogs for characterization and labeling of the plasma membrane $\mathrm{Na}+/ \mathrm{H}+$ antiporter from Dunaliella salina. Biochemistry. 1994;33(9):2389-93.

8. Katz A, Waridel P, Shevchenko A, Pick U. Salt-induced changes in the plasma membrane proteome of the halotolerant alga Dunaliella salina as revealed by blue native gel electrophoresis and nano-LC-MS/MS analysis. Mol Cell Proteomics. 2007;6(9):1459-72.

9. Liska AJ, Shevchenko A, Pick U, Katz A. Enhanced photosynthesis and redox energy production contribute to salinity tolerance in Dunaliella as revealed by homology-based proteomics. Plant Physiol. 2004;136(1):2806-17.

10. Tan KWM, Lin H, Shen H, Lee YK. Nitrogen-induced metabolic changes and molecular determinants of carbon allocation in Dunaliella tertiolecta. Sci Rep. 2016;6:37235.

11. Yao L, Tan KWM, Tan TW, Lee YK. Exploring the transcriptome of non-model oleaginous microalga Dunaliella tertiolecta through high-throughput sequencing and high performance computing. BMC Bioinformatics. 2017; 18(1):122.

12. Yao L, Tan TW, Ng Y-K, Ban KHK, Shen H, Lin H, Lee YK. RNA-Seq transcriptomic analysis with Bag2D software identifies key pathways enhancing lipid yield in a high lipid-producing mutant of the non-model green alga Dunaliella tertiolecta. Biotechnology for Biofuels. 2015;8(1):191.

13. Hong L, Liu J-I, Midoun SZ, Miller PC. Transcriptome sequencing and annotation of the halophytic microalga Dunaliella salina. J Zhejiang Univ Sci B. 2017:18(10):833-44.

14. Alkayal F, Albion RL, Tillett RL, Hathwaik LT, Lemos MS, Cushman JC. Expressed sequence tag (EST) profiling in hyper saline shocked Dunaliella salina reveals high expression of protein synthetic apparatus components. Plant Sci. 2010;179(5):437-49.

15. Chitlaru E, Pick U. Selection and characterization of Dunaliella salina mutants defective in haloadaptation. Plant Physiol. 1989:91(2):788-94.

16. Oren A. A hundred years of Dunaliella research: 1905-2005. Saline Systems. 2005;1(1):2.

17. Polle JEW, Barry K, Cushman J, Schmutz J, Tran D, Hathwaik LT, Yim WC, Jenkins J, McKie-Krisberg Z, Prochnik S, et al. Draft Nuclear Genome Sequence of the Halophilic and Beta-Carotene-Accumulating Green Alga Dunaliella salina Strain CCAP19/18. Genome Announc. 2017;5(43):e01105-17.

18. Merchant SS, Prochnik SE, Vallon O, Harris EH, Karpowicz SJ, Witman GB, Terry A, Salamov A, Fritz-Laylin LK, Marechal-Drouard L, et al. The Chlamydomonas genome reveals the evolution of key animal and plant functions. Science (New York, NY). 2007;318(5848):245-50.

19. Terry MJ, Smith AG. A model for tetrapyrrole synthesis as the primary mechanism for plastid-to-nucleus signaling during chloroplast biogenesis. Front Plant Sci. 2013;4:14.

20. Brzezowski P, Richter AS, Grimm B. Regulation and function of tetrapyrrole biosynthesis in plants and algae. Biochim Biophys Acta. 2015;1847(9):968-85.

21. Zhang ZW, Zhang GC, Zhu F, Zhang DW, Yuan S. The roles of tetrapyrroles in plastid retrograde signaling and tolerance to environmental stresses. Planta. 2015;242(6):1263-76.

22. Voss B, Meinecke L, Kurz T, Al-Babili S, Beck CF, Hess WR. Hemin and magnesium-protoporphyrin IX induce global changes in gene expression in Chlamydomonas reinhardtii. Plant Physiol. 2011;155(2):892-905.
23. Enami K, Ozawa T, Motohashi N, Nakamura M, Tanaka K, Hanaoka M. Plastidto-nucleus retrograde signals are essential for the expression of nuclear starch biosynthesis genes during amyloplast differentiation in tobacco BY-2 cultured cells. Plant Physiol. 2011;157(1):518-30.

24. Nagahatenna DS, Langridge $P$, Whitford R. Tetrapyrrole-based drought stress signalling. Plant Biotechnol J. 2015;13(4):447-59.

25. Anjum NA, Sharma P, Gill SS, Hasanuzzaman M, Khan EA, Kachhap K, Mohamed AA, Thangavel P, Devi GD, Vasudhevan P, et al. Catalase and ascorbate peroxidase-representative $\mathrm{H} 2 \mathrm{O} 2$-detoxifying heme enzymes in plants. Environ Sci Pollut Res Int. 2016;23(19):19002-29.

26. Delorge I, Janiak M, Carpentier S, Van Dijck P. Fine tuning of trehalose biosynthesis and hydrolysis as novel tools for the generation of abiotic stress tolerant plants. Front Plant Sci. 2014;5:147.

27. He Q, Toh JD, Ero R, Qiao Z, Kumar V, Serra A, Tan J, Sze SK, Gao YG. The unusual di-domain structure of Dunaliella salina glycerol-3-phosphate dehydrogenase enables direct conversion of dihydroxyacetone phosphate to glycerol. Plant J. 2019. https://doi.org/10.1111/tpj.14619. [Epub ahead of print]

28. Azachi M, Sadka A, Fisher M, Goldshlag P, Gokhman I, Zamir A. Salt induction of fatty acid elongase and membrane lipid modifications in the extreme halotolerant alga Dunaliella salina. Plant Physiol. 2002;129(3):1320-9.

29. Schulze H, Kolter T, Sandhoff K. Principles of lysosomal membrane degradation: cellular topology and biochemistry of lysosomal lipid degradation. Biochim Biophys Acta. 2009;1793(4):674-83.

30. Hildebrandt TM, Nunes Nesi A, Araujo WL, Braun HP. Amino acid catabolism in plants. Mol Plant. 2015;8(11):1563-79.

31. Gu J, Xia Z, Luo Y, Jiang X, Qian B, Xie H, Zhu JK, Xiong L, Zhu J, Wang ZY. Spliceosomal protein U1A is involved in alternative splicing and salt stress tolerance in Arabidopsis thaliana. Nucleic Acids Res. 2018;46(4):1777-92.

32. Ding F, Cui P, Wang Z, Zhang S, Ali S, Xiong L. Genome-wide analysis of alternative splicing of pre-mRNA under salt stress in Arabidopsis. BMC Genomics. 2014;15:431.

33. Sampuda KM, Riley M, Boyd L. Stress induced nuclear granules form in response to accumulation of misfolded proteins in Caenorhabditis elegans. BMC Cell Biol. 2017;18(1):18.

34. Kosova K, Vitamvas P, Prasil IT. Proteomics of stress responses in wheat and barley-search for potential protein markers of stress tolerance. Front Plant Sci. 2014:5:711.

35. Ben-Amotz A, Sussman I, Avron M. Glycerol production by Dunaliella. In: New Trends in Research and Utilization of Solar Energy through Biological Systems: Springer; 1982. p. 55-8.

36. Catterall WA. From ionic currents to molecular mechanisms: the structure and function of voltage-gated sodium channels. Neuron. 2000;26(1):13-25.

37. Rocher A, Obeso A, Cachero MT, Herreros B, Gonzalez C. Participation of $\mathrm{Na}+$ channels in the response of carotid body chemoreceptor cells to hypoxia. Am J Phys. 1994;267(3 Pt 1):C738-44.

38. Lopez-Lopez J, Gonzalez C, Urena J, Lopez-Barneo J. Low pO2 selectively inhibits K channel activity in chemoreceptor cells of the mammalian carotid body. J Gen Physiol. 1989;93(5):1001-15.

39. Moriguchi T, Urushiyama S, Hisamoto N, lemura S, Uchida S, Natsume T, Matsumoto K, Shibuya H. WNK1 regulates phosphorylation of cationchloride-coupled cotransporters via the STE20-related kinases, SPAK and OSR1. J Biol Chem. 2005;280(52):42685-93.

40. Ponce-Coria J, San-Cristobal P, Kahle KT, Vazquez N, Pacheco-Alvarez D, de Los HP, Juarez P, Munoz E, Michel G, Bobadilla NA, et al. Regulation of NKCC2 by a chloride-sensing mechanism involving the WNK3 and SPAK kinases. Proc Natl Acad Sci U S A. 2008;105(24):8458-63.

41. Ring AM, Cheng SX, Leng Q, Kahle KT, Rinehart J, Lalioti MD, Volkman HM, Wilson FH, Hebert SC, Lifton RP. WNK4 regulates activity of the epithelial Na+ channel in vitro and in vivo. Proc Natl Acad Sci U S A. 2007;104(10):4020-4.

42. Xu BE, Stippec S, Lazrak A, Huang CL, Cobb MH. WNK1 activates SGK1 by a phosphatidylinositol 3-kinase-dependent and non-catalytic mechanism. J Biol Chem. 2005;280(40):34218-23.

43. Wang HR, Liu Z, Huang CL. Domains of WNK1 kinase in the regulation of ROMK1. Am J Physiol Renal Physiol. 2008;295(2):F438-45.

44. Kahle KT, Wilson FH, Leng Q, Lalioti MD, O'Connell AD, Dong K, Rapson AK, MacGregor GG, Giebisch G, Hebert SC, et al. WNK4 regulates the balance between renal $\mathrm{NaCl}$ reabsorption and K+ secretion. Nat Genet. 2003;35(4):372-6.

45. Sukharev SI, Martinac B, Arshavsky W, Kung C. Two types of mechanosensitive channels in the Escherichia coli cell envelope: solubilization and functional reconstitution. Biophys J. 1993;65(1):177-83. 
46. Haswell ES, Phillips R, Rees DC. Mechanosensitive channels: what can they do and how do they do it? Structure. 2011;19(10):1356-69.

47. Jimenez C, Berl T, Rivard CJ, Edelstein CL, Capasso JM. Phosphorylation of MAP kinase-like proteins mediate the response of the halotolerant alga Dunaliella viridis to hypertonic shock. Biochim Biophys Acta. 2004;1644(1): 61-9.

48. Gu K, Chiam H, Tian D, Yin Z. Molecular cloning and expression of heteromeric ACCase subunit genes from Jatropha curcas. Plant Sci. 2011; 180(4):642-9.

49. Goyal A. Osmoregulation in Dunaliella, part II: photosynthesis and starch contribute carbon for glycerol synthesis during a salt stress in Dunaliella tertiolecta. Plant Physiol biochem. 2007;45(9):705-10.

50. Ramos AA, Polle J, Tran D, Cushman JC, Jin E-S, JCJA V. The unicellular green alga Dunaliella salina Teod as a model for abiotic stress tolerance: genetic advances and future perspectives. Algae. 2011;26(1):3-20.

51. Chitlaru E, Pick U. Regulation of glycerol synthesis in response to osmotic changes in dunaliella. Plant Physiol. 1991;96(1):50-60.

52. Katz A, Pick U, Avron M. Modulation of $\mathrm{Na} / \mathrm{H}$ Antiporter activity by extreme pH and salt in the Halotolerant alga Dunaliella salina. Plant Physiol. 1992; 100(3):1224-9.

53. Pick U, Karni L, Avron M. Determination of ion content and ion fluxes in the halotolerant alga Dunaliella salina. Plant Physiol. 1986;81(1):92-6.

54. Hackl T, Hedrich R, Schultz J, Forster F. Proovread: large-scale high-accuracy PacBio correction through iterative short read consensus. Bioinformatics. 2014;30(21):3004-11.

55. Iseli C. ESTScan: a program for detecting, evaluating, and reconstructing potential coding regions in EST sequences. Proc Int Conf Intell Syst Mol Biol. 1999;138-48.

56. Anders S, Pyl PT, Huber W. HTSeq--a Python framework to work with highthroughput sequencing data. Bioinformatics. 2015;31(2):166-9.

57. Trapnell C, Williams BA, Pertea G, Mortazavi A, Kwan G, van Baren MJ, Salzberg SL, Wold BJ, Pachter L. Transcript assembly and quantification by RNA-Seq reveals unannotated transcripts and isoform switching during cell differentiation. Nat Biotechnol. 2010;28(5):511-5.

58. Kohonen T. The self-organizing map. J Proceedings of the IEEE. 1990;78(9): 1464-80.

59. Anders S, Huber W. Differential expression analysis for sequence count data. Genome Biol. 2010;11(10):R106.

60. Conesa A, Gotz S, Garcia-Gomez JM, Terol J, Talon M, Robles M. Blast2GO: a universal tool for annotation, visualization and analysis in functional genomics research. Bioinformatics. 2005;21(18):3674-6.

61. Young MD, Wakefield MJ, Smyth GK, Oshlack A. Gene ontology analysis for RNA-seq: accounting for selection bias. Genome Biol. 2010;11(2):R14.

62. Mao X, Cai T, Olyarchuk JG, Wei L. Automated genome annotation and pathway identification using the KEGG Orthology (KO) as a controlled vocabulary. Bioinformatics. 2005;21(19):3787-93.

\section{Publisher's Note}

Springer Nature remains neutral with regard to jurisdictional claims in published maps and institutional affiliations.

Ready to submit your research? Choose BMC and benefit from:

- fast, convenient online submission

- thorough peer review by experienced researchers in your field

- rapid publication on acceptance

- support for research data, including large and complex data types

- gold Open Access which fosters wider collaboration and increased citations

- maximum visibility for your research: over $100 \mathrm{M}$ website views per year

At BMC, research is always in progress.

Learn more biomedcentral.com/submissions 